\title{
Path Loss Models and Large Scale Fading Statistics for C-Band Train-to-Train Communication
}

\author{
Paul Unterhuber*, Ibrahim Rashdan*, Michael Walter*, Thomas Kürner ${ }^{\dagger}$ \\ ${ }^{*}$ Institute of Communications and Navigation, German Aerospace Center (DLR), Oberpfaffenhofen, 82234 Wessling, \\ Germany, paul.unterhuber@dlr.de \\ ${ }^{\dagger}$ Institute for Communications Technology, Technische Universität Braunschweig, 38106 Braunschweig, Germany
}

\begin{abstract}
The profound knowledge of wireless propagation is essential for wireless communication between vehicles. To evolve and test communication standards we need channel models in representative environments to neither over-, nor underestimate the effect of the surrounding environment and the movement of the vehicles; typical environments for railway communication are railway station, open field and hilly environments.

We introduce train-to-train (T2T) path loss models and large scale fading statistics based on channel sounder measurement data as a first step towards a geometry-based stochastic channel model (GSCM). The models represent the mentioned typical environments for railway applications. We compare the results with previous published intelligent transportation system (ITS-G5) measurement based models and highlight the differences.
\end{abstract}

Index Terms - train-to-train, high speed train, propagation, path loss models, fading statistics.

\section{INTRODUCTION}

Reliable train-to-train (T2T) communication is the key technology for next generation of railway applications and control, and will enable virtual coupled and autonomously driving high speed trains [1]. Several projects and research groups are focusing on T2T communication like Roll2Rail and X2Rail within the Shift2Rail initiative [2]. [3] focuses on intelligent transportation system (ITS-G5) within the Secure Connected Trustable Thing (SCOTT) project [4], or [5] by adopting Long Term Evolution - vehicle to everything (LTE-V2X) to the needs of railway applications.

The basis of reliable communication is the knowledge of the influence of the propagation channel on the wireless transmission. Therefore, we performed a comprehensive measurement campaign within the Roll2Rail project with two high speed trains and the DLR RUSK channel sounder [6]. The large measurement bandwidth and high spatial resolution of the sounding measurements allow a detailed analysis of the propagation characteristics. The data set is divided in railway station, open field and hilly environment and path loss models and large scale fading statistics are derived. We compare the derived parameters with existing T2T channel models presented in [7]. These previous presented path loss (PL) models are based on received signal strength indication (RSSI) measurements with ITS-G5 transceivers. The new models are based on more extensive and preciser measurement data and therefore sharpen and extend the existing models.
In a future step, the proposed path loss models will be embedded into a geometry-based stochastic channel model (GSCM) for T2T.

\section{Channel Sounding Measurement Campaign}

\section{A. Measurement Equipment and Setup}

The channel sounding measurements were performed with the DLR RUSK channel sounder. The sounder consists of rubidium frequency standards at transmitter (Tx) and receiver (Rx) side and was operated in single-input single-output (SISO) mode with omni-directional railway antennas at $f=$ $5.2 \mathrm{GHz}$ and a bandwidth of $120 \mathrm{MHz}$. The measurements were performed with a snapshot rate of $1.024 \mathrm{~ms}$ and a maximum excess delay of $12.8 \mu \mathrm{s}$.

The two trains were driving on two parallel tacks for safety reason. Nevertheless, we could perform approaching or separation maneuvers as for the virtual coupling application with different velocities. The analyzed link distances reaches from $10 \mathrm{~m}$ to $1 \mathrm{~km}$. The position and velocity of the trains were tracked with multiple global navigation satellite system (GNSS) receivers in both trains. Further details about the channel sounder can be found in [6] and the measurement campaign in [8].

\section{B. Environment and Track Architecture}

In this paper we focus only on the path loss models and large scale fading for T2T communications. Nevertheless, for the proposed models we analyzed the environment and the architecture of the track. The high speed track between the station Roma Termini and station Napoli Centrale has a total length of $204.6 \mathrm{~km}$. In total $39 \mathrm{~km}$ are tunnels, where the longest tunnel is $6725 \mathrm{~m}$; viaducts and bridges cover $39 \mathrm{~km}$. The biggest share of the track runs through open field and hilly environment [9]. Hence, we differentiate between railway station, open field and hilly environments and split all evaluations and the resulting models into these three categories. Tunnels are not covered within this contribution.

The track architecture has a significant influence on the wireless propagation. For distances up to $1 \mathrm{~km}$ obstacles along the track like overhead line masts, cross bridges or other trains cause MPCs as shown in the top view of Fig. 1, or may cause obstructed LOS (OLOS) in curved track segments. The overhead line masts have a maximum spacing of $60 \mathrm{~m}$, cross bridges and other trains occur randomly. By increasing the 


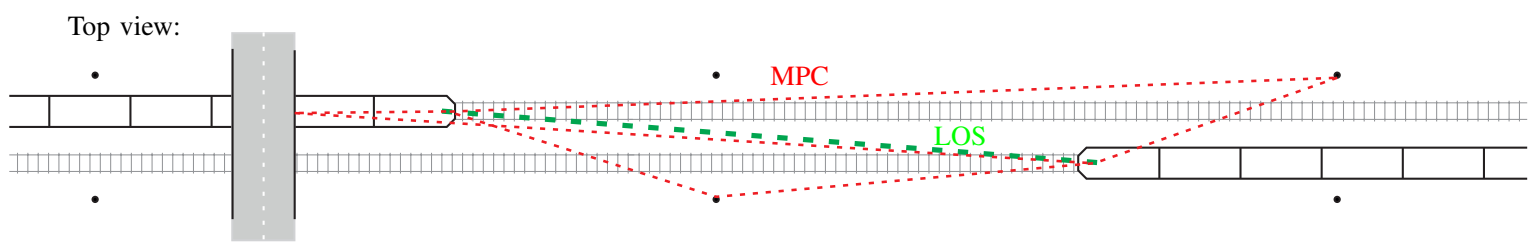

$\mathrm{Tx}$

$\mathrm{Rx}$

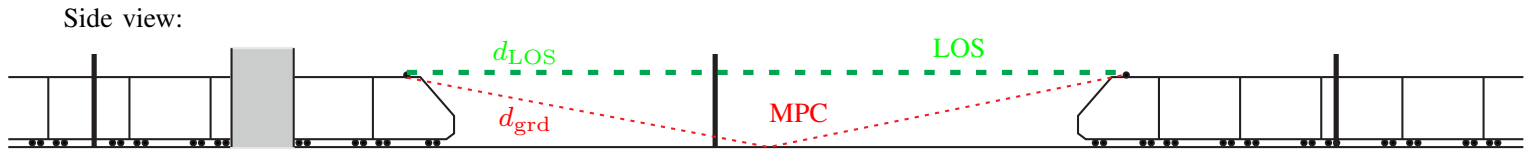

Fig. 1. LOS and multipath components (MPCs) caused by overhead line masts, cross bridge and ground reflection for T2T communication.

distance to several kilometers non LOS (NLOS) may occur in combination with large buildings, cuttings and hills.

\section{PATH LOSS MODELS}

In this section we derive power delay profiles (PDPs) from the measurement data for three environments. Due to the complex measurement setup with two high speed trains, measurement data of only one run for each environment is available. The instantaneous PDP gives us a good overview of the richness of the fading behavior and appearing MPCs. The probability density function (PDF) of the normalized PDP shows the probability of the received power and the large scale fading behavior. Finally, based on the PDP we derive the PL over distance, fit different PL models and derive large scale fading statistics.

\section{A. Power Delay Profiles}

The measured channel impulse response (CIR) $h(t, \tau)$ at the time instant $t$ and delay $\tau$ can be modeled as a sum of Dirac impulses

$$
h(t, \tau)=\sum_{i=0}^{N} \alpha_{i}(t) \cdot \delta_{i}\left(\tau-\tau_{i}\right),
$$

where $\alpha_{i}$ stands for the complex amplitude and $\tau_{i}$ for the delay of the $i$ th path. The instantaneous PDP is derived as [10]

$$
\operatorname{PDP}(t, \tau)=|h(t, \tau)|^{2}
$$

and plotted for three environments in Fig. 2. In Fig. 2a we see a configuration with standing Tx and Rx till $t=20 \mathrm{~s}$. After, the Tx start moving while the Rx remains at the platform at station Napoli Centrale. The PDP of an overtaking maneuver in open field environment is shown in Fig. 2b; during the time of 75 $87 \mathrm{~s}$ interpolated data leads to a blurry area because a new measurement was started. The PDP of the hilly environment is plotted in Fig. 2c. A line of sight (LOS) reference based on GNSS distance is plotted in red in all figures.

In all three PDP plots we clearly see regular patterns of MPCs. The richest MPCs can be found in the railway station environment. Platform roofs, other trains, masts and signs scatter the sounding signal. Up from $t=40 \mathrm{~s}$ the MPCs appear in a regular spacing in the same way as for the other two environments. These regular appearing MPCs are mainly caused by overhead line masts. If we analyze the spacing between the MPCs in Fig. 2b in the delay domain, we can derive a spacing of $55 \mathrm{~m}$ to $59 \mathrm{~m}$. This goes along with the standard spacing of overhead line masts of maximum $60 \mathrm{~m}$.

Due to the continuous movement of both, Tx and Rx the wide sense stationarity assumption does not hold. We assume stationarity within a minimum stationarity time $t_{\text {stat }}=\frac{w \cdot \lambda}{\bar{v}}$ for all measurements and the corresponding average velocity $\bar{v}$. Hence, the averaged PDP is derived by applying a moving averaging function with a window length $t_{\text {stat }}$, were $w=10$ was the best fit to our data. Nevertheless, we will investigate a time varying $t_{\text {stat }}$ as performed in [11]. Within $t_{\text {stat }}$ we remove the small scale fading and derive $\overline{P D P(t, \tau)}$. Finally, the received power can be expressed as the sum over $\tau$ for all MPCs as

$$
P_{R x}(t)=\sum_{i=0}^{N} \overline{P D P\left(t, \tau_{i}\right)}
$$

For evaluating the power distribution, we normalize the delay and power of the averaged PDP to the LOS delay and free-space path loss (FSPL). Furthermore, we summed up the normalized PDPs for measurements in the same environment and plotted the resulting PDFs in Fig. 3. As for the instantaneous PDP also the normalized PDP for the railway station environment shows the most large scale fading for short link distances up to $225 \mathrm{~m}$ or $0.75 \mu \mathrm{s}$. Up to the mentioned delay we clearly see several spikes in Fig. 3a. The probability shows a clear concentration of the power for MPCs with low delay smaller than $1 \mu \mathrm{s}$.

In comparison, the open field environment shows only one spike at $0.19 \mu$ s with a power of $-34.6 \mathrm{~dB}$. For higher delays the power drops very fast below $-40 \mathrm{~dB}$ for $\tau>0.37 \mu \mathrm{s}$. Nevertheless, the power is distributed over a larger delay scale. A similar spike can be also observed for the hilly environment at $0.19 \mu$ s with a power of $-34.9 \mathrm{~dB}$, but the power for higher delays drops slower below $-40 \mathrm{~dB}$ for $\tau>0.97 \mu \mathrm{s}$ as for the other environments. Similar as for the railway station, the probability shows a concentration on short MPCs.

As this one spike appears at a constant $\tau=0.19 \mu$ s in open field and hilly environment the causing reflector need to be an object on one of the trains, e.g. pantograph or antennas. 


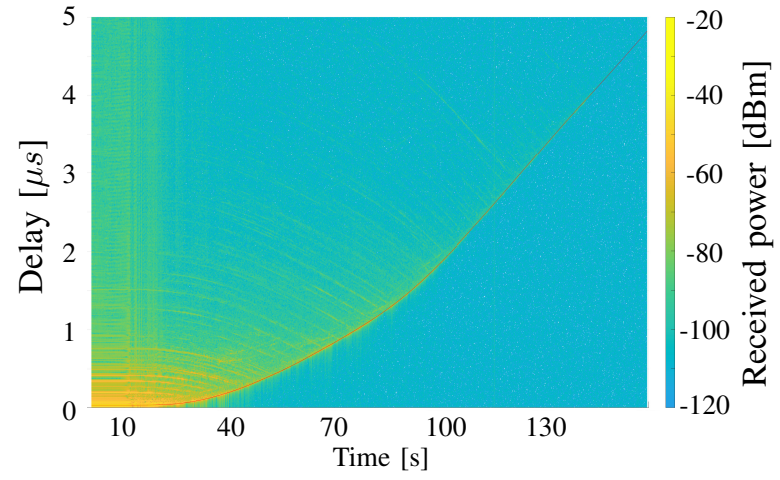

(a) Railway station.

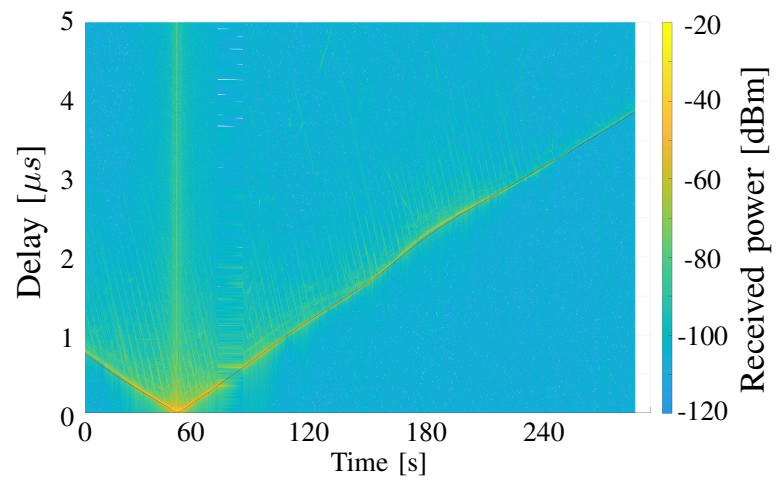

(b) Open field.

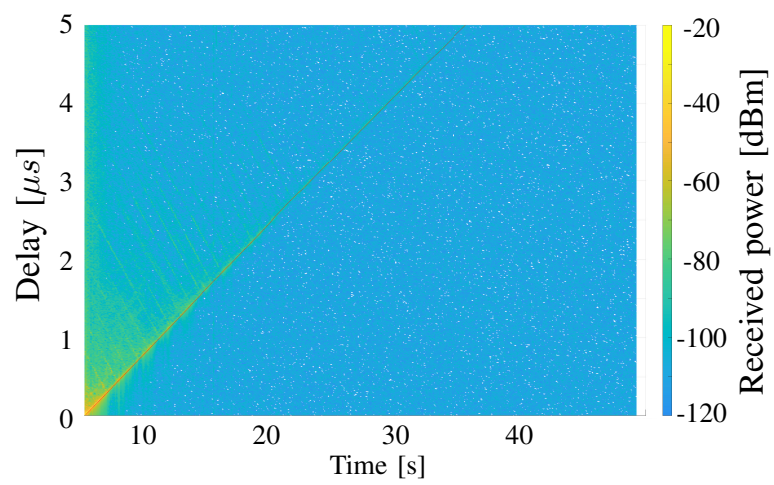

(c) Hilly with cuttings.

Fig. 2. Instantaneous PDPs and GNSS based LOS distance as reference (red solid line) for railway environments.

\section{B. Free Space Path Loss}

Based on the relation between the received power $P_{\mathrm{Rx}}$ and the transmitted power $P_{\mathrm{Tx}}$ with respect to the gains of the Tx amplifier, cables and antenna $G_{\mathrm{Tx}}$ and Rx low noise amplifier, cables and antenna $G_{\mathrm{Rx}}$ the path loss can be expressed as

$$
P L(t)=\frac{P_{\mathrm{Tx}}(t)}{P_{\mathrm{Rx}}(t)} \cdot G_{\mathrm{Tx}} G_{\mathrm{Rx}} \cdot
$$

Resolving the PL based on the distance $d$ we can write

$$
P L(d)=10 n \cdot \log \left(\frac{4 \pi \cdot d \cdot f}{c}\right),
$$

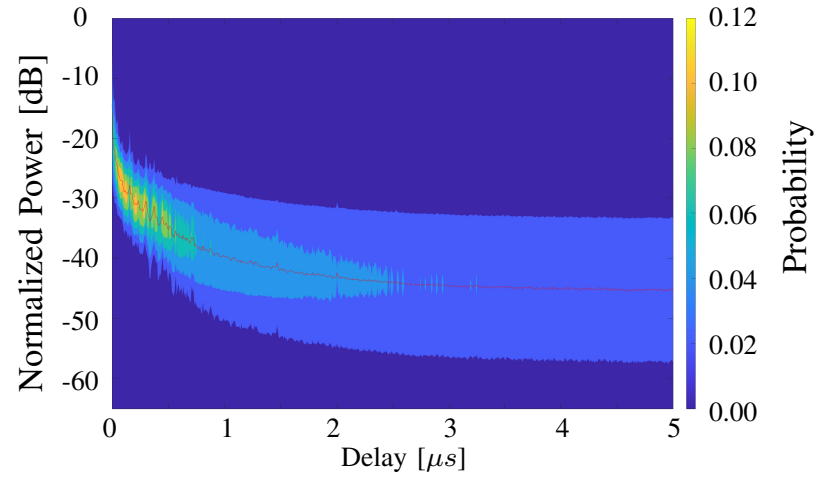

(a) Railway station.

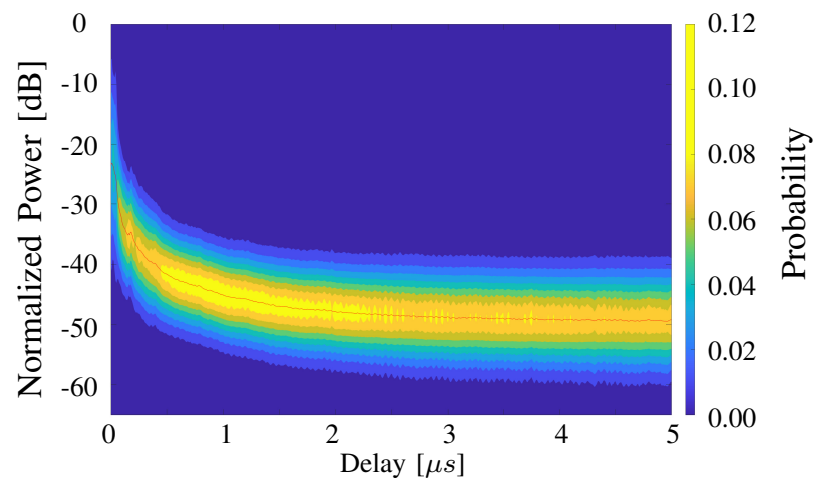

(b) Open field.

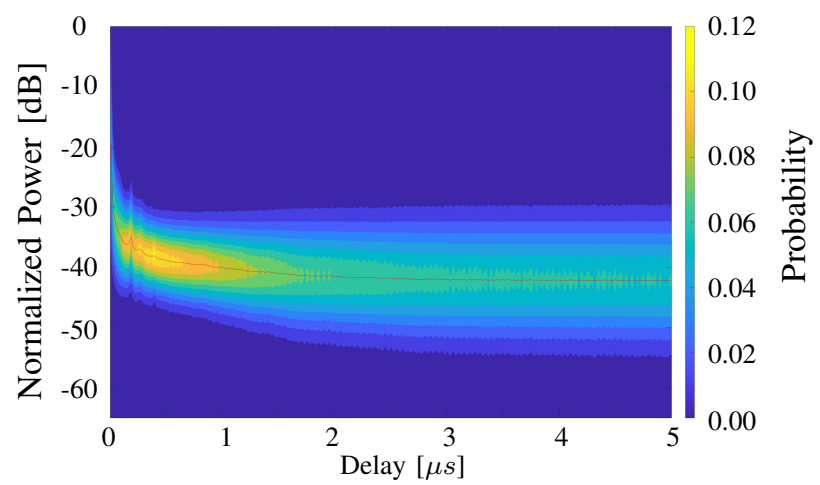

(c) Hilly with cuttings.

Fig. 3. PDFs of the normalized PDPs and the mean normalized PDP (red solid line) for railway environments.

where $f$ denotes the frequency and $c$ the speed of light. For $n=2$ we obtain the FSPL which is indicated as red dashed line in all three sub-figures of Fig. 4.

\section{Log-Distance Path Loss}

Up from a certain link distance all of our measurements show a mixture of LOS and OLOS situations with shadow fading. Hence, the log-distance path loss model with lognormal shadowing was applied. In (6) the total path loss $P L(d)$ is composed of a path loss $P L\left(d_{0}\right)$ at a fixed reference distance $d_{0}, n$ times the logarithm of the relation between the 


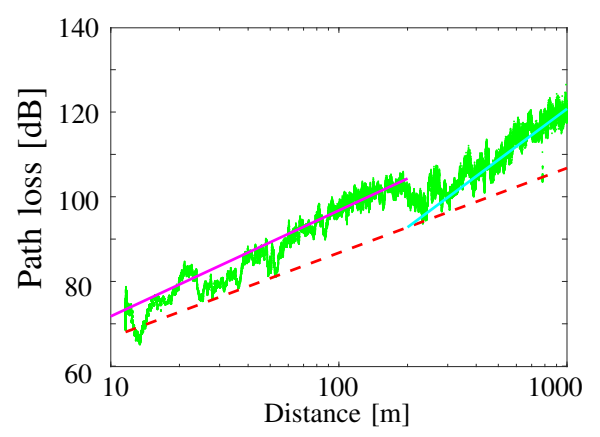

(a) Railway stataion.

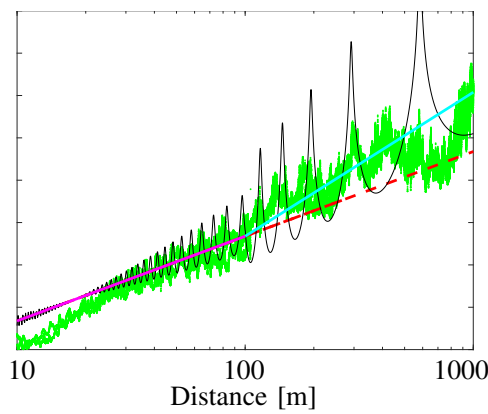

(b) Open field.

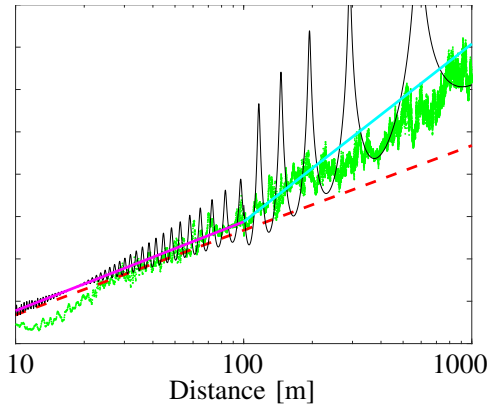

(c) Hilly with cuttings.

Fig. 4. PL for railway environments: the green dots represent the PL from measurement data, the red dashed line marks the FSPL, the magenta and blue lines shows the two-slope log-distance PL and the the black line indicates the two-ray PL.

TABLE I

TWO-SLOPE LOG-DISTANCE PL MODEL PARAMETERS IN COMPARISON WITH THE RESULTS OF [7].

\begin{tabular}{|c|c|c|c|c|c|c|c|c|}
\hline & \multicolumn{2}{|c|}{$d_{0}[\mathrm{~m}]$} & \multicolumn{2}{c|}{$P_{0}[\mathrm{dBm}]$} & \multicolumn{2}{c|}{$n$} & \multicolumn{2}{c|}{$\sigma$ [dB] } \\
Slope & 1 & 2 & 1 & 2 & 1 & 2 & 1 & 2 \\
\hline Railway station & 1 & 200 & 46.768 & 92.788 & 2.5 & 4 & 2.622 & 2.894 \\
Open field & 1 & 100 & 46.768 & 86.768 & 2 & 3.5 & 2.907 & 5.661 \\
Hilly with cutting & 1 & 100 & 46.768 & 86.768 & 2.1 & 4.2 & 2.930 & 3.914 \\
\hline Sub-urban [7] & 10 & 400 & 47.86 & 60.36 & 3 & 2.15 & 4.95 & 4.96 \\
Rural [7] & 1 & - & 47.86 & - & 2.14 & - & 4.22 & - \\
Tunnel [7] & 80 & 800 & 59.42 & 101.42 & 4.15 & 1.85 & 5.01 & 6.96 \\
\hline
\end{tabular}

actual link distance $d$ and $d_{0}$ and a Gaussian distributed vector $\chi_{\sigma} \sim \mathcal{N}\left(0, \sigma^{2}\right)$ with a standard deviation $\sigma[12]$.

$$
P L(d)=P L\left(d_{0}\right)+10 n \cdot \log \left(\frac{d}{d_{0}}\right)+\chi_{\sigma}
$$

In a railway station environment, the PL for distances below $200 \mathrm{~m}$ is highly attenuated in comparison to the FSPL with deep fades up to $10 \mathrm{~dB}$ as shown in Fig. 4a. On the first $80 \mathrm{~m}$ the OLOS is caused by shadowing from the platform roof between the two trains and up to $200 \mathrm{~m}$ due to the dense infrastructure around the tracks. This behavior could be observed for train-to-ground communication by [13] as well The section up to $200 \mathrm{~m}$ is modeled with the first slope of the log-distance PL model with a path loss exponent $n=2.5$ and a standard deviation $\sigma=2.622 \mathrm{~dB}$. At $200 \mathrm{~m}$ the first driving train switches the track and continues in a wide open station or shunting yard similar environment right in front of the second train which is still standing in the station; this change can be observed in the measurements as deep fades and is modeled as the second slope with $n=4$ and $\sigma=2.894 \mathrm{~dB}$.

The second interesting environment is shown in Fig. 4b. In open field the log-distance model follows the FSPL up to a distance of $100 \mathrm{~m}$ with $n=2$ and $\sigma=2.907 \mathrm{~dB}$. For larger distances the path loss exponent changes to $n=3.5$ and the standard deviation to $\sigma=5.661 \mathrm{~dB}$. This is mainly caused by the influence of the ground reflection.

A similar, but slightly worse behavior as in open field can be observed in a hilly environment with cuttings in Fig. 4c. Within the first stage of the model up to $100 \mathrm{~m}, n=2.1$ as for
FSPL with $\sigma=2.93 \mathrm{~dB}$. For higher distances the parameters of the second slope changes to $n=4.2$ and $\sigma=3.914 \mathrm{~dB}$. It is clearly visible, that the simple two-ray PL is not sufficient for the richness of MPCs in the hilly environment. In Table I the log-distance PL model parameters are summarized.

\section{Two-Ray Path Loss}

The two-ray PL model is taken into account the fading effect of the ground reflection as shown in the side view of Figure 1. The following approach was presented for mobile communication in [14]:

$$
\begin{aligned}
P L(d)= & 20 \log \left(\frac{4 \pi \cdot f}{c}\right)- \\
& 20 \log \left|\frac{\mathrm{e}^{-\mathrm{j} \frac{2 \pi \cdot f}{c} d_{\mathrm{LOS}}}}{d_{\mathrm{LOS}}}+\Gamma(\Theta) \frac{\mathrm{e}^{-\mathrm{j} \frac{2 \pi \cdot f}{c} d_{\mathrm{grd}}}}{d_{\text {grd }}}\right| .
\end{aligned}
$$

The ground reflection coefficient $\Gamma(\Theta)$ is defined as

$$
\left.\Gamma(\Theta)=\frac{\epsilon_{\mathrm{r}} \sin (\Theta)-\sqrt{\epsilon_{\mathrm{r}}-\cos ^{2}(\Theta)}}{\epsilon_{\mathrm{r}} \sin (\Theta)+\sqrt{\epsilon_{\mathrm{r}}-\cos ^{2}(\Theta)}}\right),
$$

with the reflection angle $\Theta$ and the relative permittivity $\epsilon_{\mathrm{r}}$ of the ground surface [12].

The two-ray PL model and the log-distance PL model were combined to take into account the ground reflection with respect to the different slopes.

$$
\begin{aligned}
P L(d)= & P L\left(d_{0}\right)+10 n \cdot \log \left(\frac{1}{d_{0}}\right)- \\
& 20 \log \left|\frac{\mathrm{e}^{-\mathrm{j} \frac{2 \pi \cdot f}{c} d_{\mathrm{LOS}}}}{d_{\mathrm{LOS}}}+\Gamma(\Theta) \frac{\mathrm{e}^{-\mathrm{j} \frac{2 \pi \cdot f}{c} d_{\mathrm{grd}}}}{d_{\mathrm{grd}}}\right| .
\end{aligned}
$$


In case of T2T communication with two trains of the same height $h_{\mathrm{Tx}}=h_{\mathrm{Rx}}=4.1 \mathrm{~m}$, the observed distance $d$ projected on the earth surface based on the GNSS data equals to the propagation distance for the LOS path $d_{\mathrm{LOS}}$. Based on investigations on the relative permittivity of railway materials in [15], $\epsilon_{\mathrm{r}}$ was set to 4.8 for granite clean ballast in dry conditions. This results in a good match of the two-ray PL to the measurement data as shown in Fig. $4 \mathrm{~b}$ for the open field and in Fig. 4c for hilly environment. For the railway station environment, this model was not suitable.

\section{Comparison to ITS-G5 Measurements}

Within this measurement campaign, measurements with ITS-G5 transceivers were performed as well as presented in [8]. Details about these measurement setup and resulting path loss models based on these ITS-G5 measurements are published in [7]. In comparison to the channel sounder CIR, the ITS-G5 transceivers record only RSSI values. The RSSI values represent an average of the received power of each received communication package.

For both, the channel sounder and ITS-G5 measurement setup we had to use the same roof mounted antenna on the $\mathrm{Tx}$ train and on the $\mathrm{Rx}$ train. Therefore, the ITS-G5 measurements were performed in night two and the channel sounder measurements in night three within the measurement campaign. Even though we used the same trains and drove on the same tracks, the maneuvers differ between night two and three slightly. Hence, PL models for rural, sub-urban and tunnels based on ITS-G5 measurements were presented in [7]. The railway station and hilly with cutting environment are hardly comparable with sub-urban and tunnels and are complimentary to the previous presented models.

Therefore, only the rural area can be compared to the open field environment. [7, Fig. 10] shows the RSSI values fitted with a single log-distance PL model with $n=2.14$ and $\sigma=4.22 \mathrm{~dB}$ for distances up to $400 \mathrm{~m}$. It can be observed, that the RSSI values for distances above $200 \mathrm{~m}$ show strong variations in the same way as in the channel sounder measurements. By applying a two-stage log-distance model to the channel sounder data as in Fig. $4 \mathrm{~b}$ instead of a one-stage as in [7] the fitted slope up to $100 \mathrm{~m}$ relaxes with $n=2$ and the second slope with $n=3.8$ for higher distances takes the strong variation better into account. Therefore, the presented open field PL model sharpen and extend the previous presented PL model. All results are summarized in Table I.

\section{CONCLUSION}

In this paper we analyzed the channel sounding data of an extensive T2T measurement campaign and derived PL models for three typical railway environments. For the railway station, open field and hilly environment with cuttings, the PDPs and PDFs of the PDPs were presented. The regular appearing patterns of MPCs can be explained by the surrounding obstacles like overhead line masts and cross bridges. Strong influences of the train body itself could be shown with the normalized PDPs. Based on the PDPs, the PL for all three environments have been derived and fitted with FSPL and logdistance PL models. For the open field and hilly environment, a two-ray PL model could be applied as well. Furthermore, we compared the new open field PL model with a previous presented model based on ITS-G5 measurements. The new two-stage log-distance PL model sharpen the model for short distances and especially in combination with the two-ray PL model, the new PL model extend the previous one for larger distances.

\section{REFERENCES}

[1] J. Moreno García-Loygorri, J. Riera, L. de Haro, and C. Rodríguez, "A Survey on Future Railway Radio Communications Services: Challenges and Opportunities," IEEE Communications Magazine, vol. 53, no. 10, pp. 62-68, Oct 2015.

[2] S2R, 2019. [Online]. Available: http://www.shift2rail.org

[3] A. A. Gómez, E. Mozo, L. Bernadó, S. Zelenbaba, T. Zemen, F. Parrilla, and A. Alberdi, "Performance analysis of its-g5 for smart train composition coupling," in 2018 16th International Conference on Intelligent Transportation Systems Telecommunications (ITST), October 2018, pp. $1-7$.

[4] Scott, "Secure Connected Trustable Thing," 2019. [Online]. Available: https://www.scottproject.eu/

[5] X. Wang, L. Liu, T. Tang, and W. Sun, "Enhancing communicationbased train control systems through train-to-train communications," IEEE Transactions on Intelligent Transportation Systems, pp. 1-18, August 2018.

[6] P. Unterhuber, S. Pfletschinger, S. Sand, M. Soliman, T. Jost, A. Arriola, I. naki Val, C. Cruces, Moreno García-Loygorri, J. GarcíaNieto, C. Rodríguez, M. Berbineau, E. Echeverría, and I. Baz, "A Survey of Channel Measurements and Models for Current and Future Railway Communication Systems," Mobile Information Systems, vol. 2016, pp. 1-14, June 2016. [Online]. Available: http://dx.doi.org/10.1155/2016/7308604

[7] P. Unterhuber, S. Sand, U.-C. Fiebig, and B. Siebler, "Path loss models for train-to-train communications in typical high speed railway environments," IET Microwaves, Antennas Propagation, vol. 12, no. 4, pp. 492-500, March 2018.

[8] P. Unterhuber, S. Sand, M. Soliman, B. Siebler, A. Lehner, T. Strang, M. d'Atri, F. Tavano, and D. Gera, "Wide Band Propagation in Trainto-Train Scenarios - Measurement Campaign and First Results," in 2017 11th European Conference on Antennas and Propagation (EuCAP), March 2017, pp. 3356-3360.

[9] Wikipedia, "Rome-Naples high-speed railway," 2018. [Online]. Available: https://en.wikipedia.org/wiki/Rome\%E2\%80\%93Naples_ high-speed_railway

[10] A. F. Molisch, "Ultra-Wide-Band Propagation Channels," Proceedings of the IEEE, vol. 97, no. 2, pp. 353-371, Feb 2009.

[11] D. Czaniera, M. Käske, G. Sommerkorn, C. Schneider, R. S. Thomä, G. Del Galdo, M. Boban, and J. Luo, "Investigation on stationarity of v2 v channels in a highway scenario," p. 5.

[12] T. S. Rappaport, Wireless Communications: Principles and Practice, ser. Prentice Hall communications engineering and emerging technologies series. Prentice Hall PTR, 2002.

[13] K. Guan, Z. Zhong, B. Ai, and T. Kürner, "Propagation measurements and analysis for train stations of high-speed railway at $930 \mathrm{mhz}$," IEEE Transactions on Vehicular Technology, vol. 63, no. 8, pp. 3499-3516, Oct 2014.

[14] H. H. Xia, H. L. Bertoni, L. R. Maciel, A. Lindsay-Stewart, and R. Rowe, "Radio propagation characteristics for line-of-sight microcellular and personal communications," vol. 41, no. 10, pp. 1439-1447, October 1993.

[15] F. De Chiara, S. Fontul, and E. Fortunato, "GPR laboratory tests for railways materials dielectric properties assessment," vol. 6 , no. 10 , pp. 9712-9728, October 2014. 